\title{
Development of RF-compressed High-throughput Femtosecond Electron Microscope
}

\author{
Shuaishuai Sun, Xiaoyi Sun, Joseph Williams and Chong-Yu Ruan \\ Michigan State University, East Lansing, Michigan, United States
}

Ultrafast electron diffraction [1] and microscopy [2] has been proven to be a powerful alternative to the free electron laser facilities for studying ultrafast phenomena in materials with a high spatiotemporal resolution. However, significant challenges exist in reaching high signal-to-noise ratio at the single-shot level with femtosecond electron pulses due to the space-charge effect at a high-charge density. In ultrafast electron diffraction (UED), several schemes were employed to side-step this deleterious effect, such as reducing the cathode-to-sample distance using the compact photo-electron gun design [1], or significantly boosting the electron pulses to the relativistic energy [3]. Alternatively, via phase-space manipulation using an RF cavity [4], the pulse duration and energy spread can be reduced without sacrificing the overall throughput of the beam system [5]. However, implementing these technologies in a traditional electron microscope setting is highly challenging due to the long flight path and multiple cross-overs; both of which heighten the space-charge effect. Designing a new electron optical system to enable high-throughput ultrafast electron microscope is a key step to realize a more flexible next-generation system capable of dealing with a wide range of material processes where a low repletion rate is necessary to access the key dynamical regimes.

Here we report the development of such new ultrafast electron microscope (UEM) that utilizes RF cavities to form the longitudinal lens system. Combing such a new optical system with the existing transverse magnetic optics, the performance in the imaging, diffraction, and spectroscopy modes of the new UEM can be improved without sacrificing the throughput based on optimizing the projection of the phase space along the respective performance figure of merit $[5,6]$. With this key concept, we demonstrated the first prototype system constructed with the optical column of a Hitachi H800 TEM and a custom $40 \mathrm{keV}$ DC photoelectron gun (Fig.1a). We show that with this new UEM setup, ultrafast imaging with $\sim 10 \mathrm{~nm}$ resolution or selected-area diffraction can be conducted with $\sim 100 \mathrm{fs}$ time resolution using up to $10^{5} \mathrm{e}^{-}$ /pulse at $1 \mathrm{kHz}$ repetition rate. We demonstrate the performance by studying the photo-induced phase transitions in the charge density wave (CDW) systems in $1 T-\mathrm{TaS}_{2}$. Exemplary dynamics are shown in Fig.1b for the sub-ps order parameter dynamics obtained using the diffraction mode, and, by switching the optics to the imaging mode, the spatial resolution was characterized by the step-edge of the supporting copper grid (Fig.1c). Accomplishing simultaneous high temporal and spatial resolution will offer the opportunities in addressing problems that are beyond the reach of current commercial UEM system. Such topics include understanding the non-equilibrium critical behavior of phase transition in isolated nanostructure that necessarily requires a long recovery time (10 ms or longer) [7], and the site-specific fieldenhanced dynamics surrounding the hot spots in plasmonic nanostructures [8].

For demonstrating the sensitivity of capturing the field effect, we investigated the evolution of laserinduced electron micro-plasma generation and the ensuing dynamics using the imaging capability of the new UEM system. In this experiment, the local plasma emission was initiated near the top surface of the copper grid. This emission dynamics were directly examined through the field-induced effects in the recorded images caused by the developing plasma (Fig.2a) with sub-ps resolution. We subsequently resolved a novel plasma echo phenomenon and its resonant coupling to the collective modes of the entire plasma fields. The system finally decays through mode coupling and settles into an upper hybrid mode at longer time $(>1 \mathrm{~ns})$. 
We acknowledge support by DOE Grant DE-FG02-06ER46309 and NSF MRI Grant DMR 1625181.

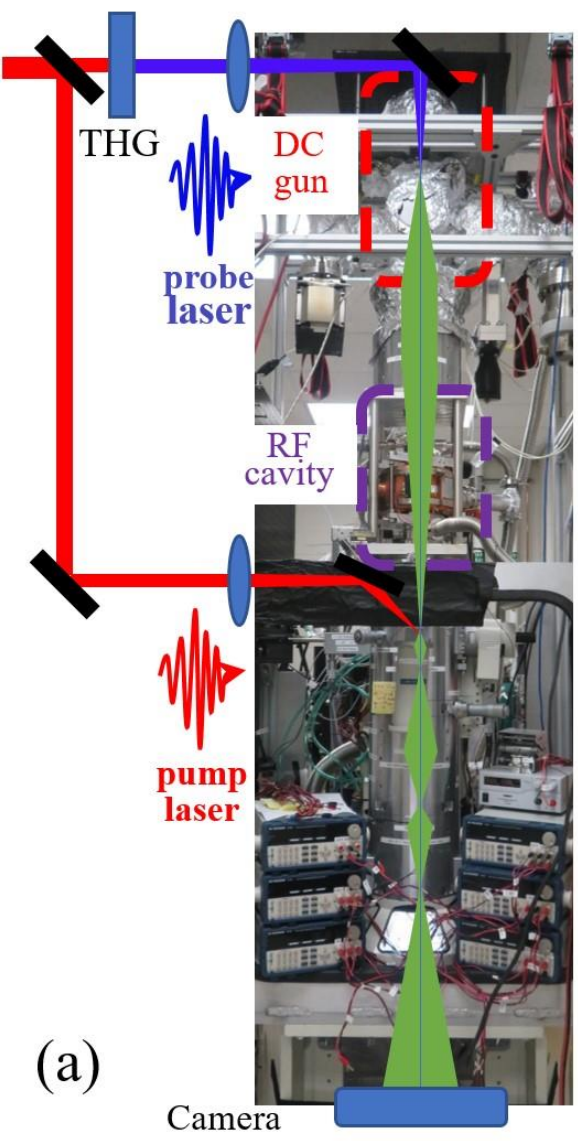

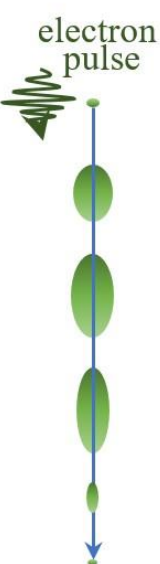

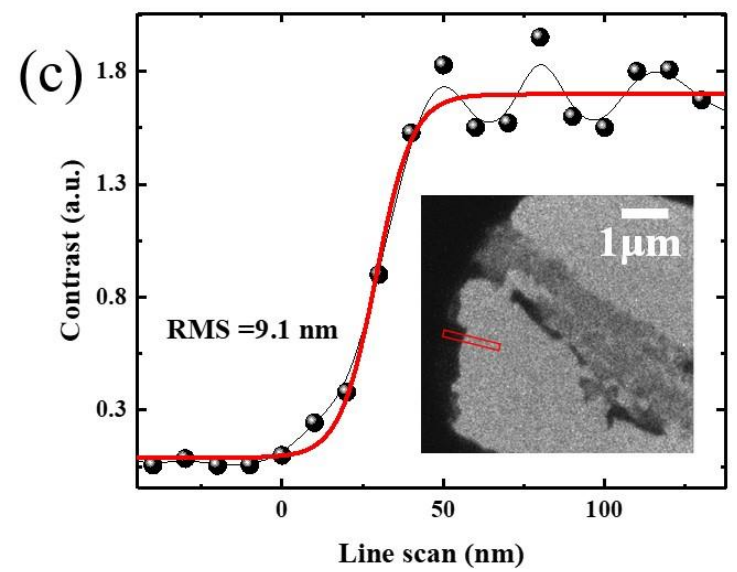

Figure 1. RF compressed UEM. (a) Setup. (b) Time resolution characterized by photo-induced CDW phase transition in 1T-TaS2. (c) Spatial resolution characterized by step-edge of the supporting copper grid along the red rectangle marked in the inset. 
(a)
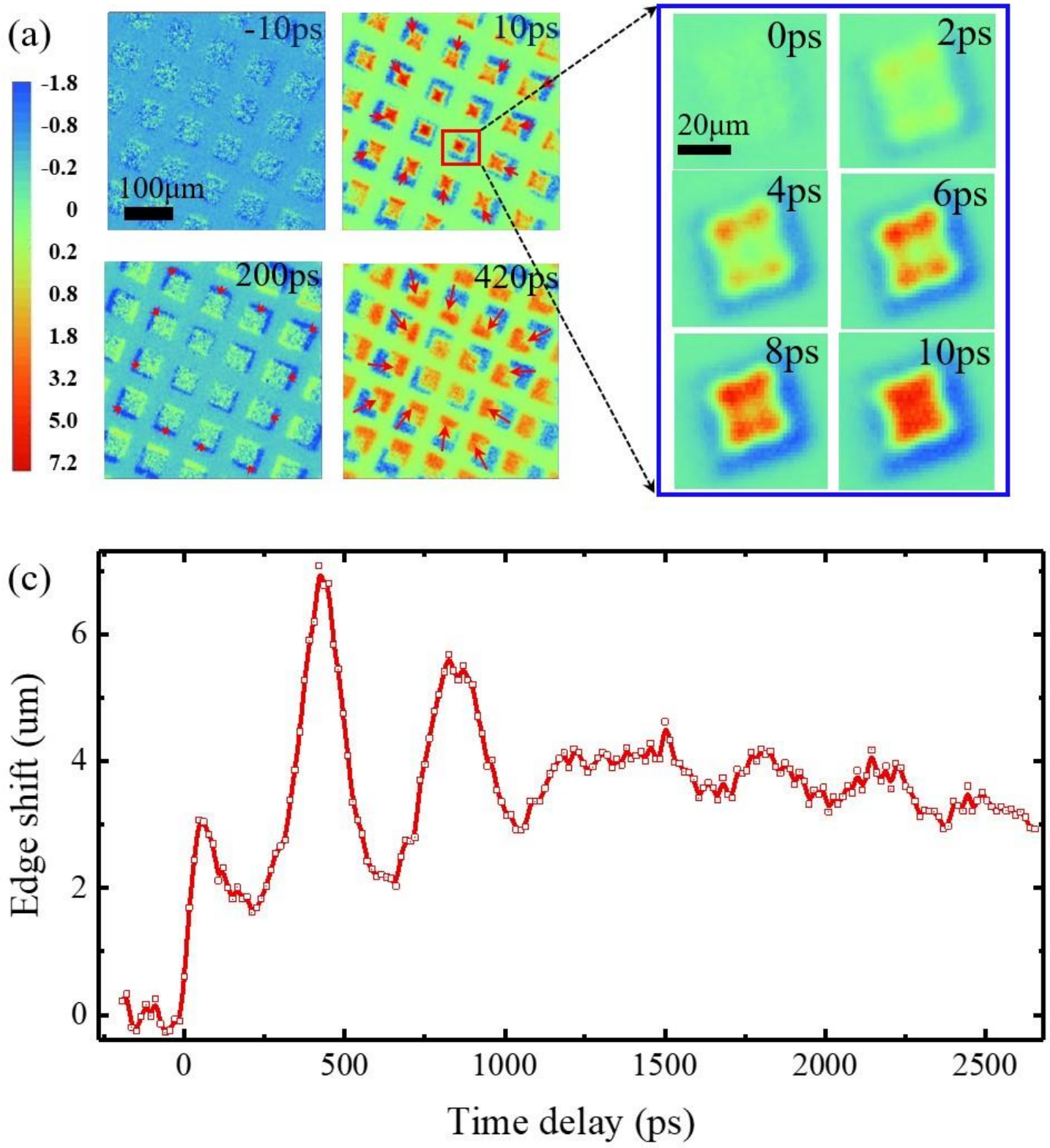

Figure 2. Laser-induced electron micro-plasma evolution. (a) Snapshot of beam deflection caused by the developing plasma field. The image is subtracted by the one taken without pump laser. Left panel has a rotation angle due to different magnification. (b) The amplitude of edge shift showing the novel plasma echo and the coupling to the collective mode.

\section{References}

[1] R. J. D. Miller, Science 343, 1108 (2014).

[2] A. H. Zewail, Science 328, 187 (2010).

[3] T. Konstantinova, L. Wu, M. Abeykoon, et al., Phys. Rev. B 99, 180102(R) (2019).

[4] T. van Oudheusden, P. L. E. M. Pasmans, S. B. van der Geer, et al., Phys. Rev. Lett. 105, 264801 (2010).

[5] J. Williams, F. Zhou, T. Sun, et al., Struct. Dyn. 4, 044035 (2017).

[6] F. Zhou, J. Williams, and C.-Y. Ruan, Chem. Phys. Lett. 683, 488 (2017). 
[7] Z. Tao, T.-R. T. Han, S. D. Mahanti, et al., Phys. Rev. Lett. 109, 166406 (2012).

[8] E. S. A. Goerlitzer, L. E. Speichermann, T. A. Mirza, et al., Nanoscale Adv. 2, 394 (2020) 